\title{
Le tournant néolibéral de la résilience ? Pratiques et formes politiques de l'opérationnalisation de la résilience à Dakar
}

\author{
The neoliberal shift of resilience? Practices and political forms of the \\ resilience operationalization in Dakar
}

\author{
Leclercq Romain ${ }^{1}$
}

${ }^{1}$ Université Paris 8, Laboratoire LAVUE (UMR 7218), romain.leclercq2@gmail.com

RÉSUMÉ. Le concept de résilience et son opérationnalisation dans les politiques internationales ont été récemment critiqués par de nombreux auteurs comme outil de l'expansion d'une forme de "gouvernementalité » néolibérale. Dans les " pays du Sud » en particulier, son application dans des politiques d'adaptation aux risques se traduirait par un transfert de la responsabilité du risque, des pouvoirs publics vers les individus et "communautés », participant ainsi de l'extension d'une rationalité de marché à l'ensemble de la vie sociale. Pourtant, peu d'études s'intéressent aux effets concrets des programmes d'adaptation et de résilience sur les populations ciblées par ces programmes.

A partir d'une ethnographie d'un programme d'adaptation du territoire et de résilience face au risque d'inondation mis en place par la Banque mondiale et l'Etat sénégalais dans la banlieue de Dakar, cet article entend revenir sur la production des transferts de responsabilité liés à l'opérationnalisation de la résilience. II montre que ces transferts s'opèrent selon des modes de représentation et des pratiques ancrées dans un contexte.

Au final, la résilience appliquée aux populations confrontées au risque d'inondation apparait bien comme la composante d'une forme de "gouvernementalité ", mais remettant l'Etat au centre de la gestion du risque, créant un régime de pouvoir s'articulant plus que ne se confondant au régime néolibéral.

ABSTRACT. The resilience concept and its operationalization within the international policies had been criticized by authors as a way to develop a neoliberal governmentality through the world. Especially in the countries of the global South, its enforcement into adaptation and risk policies would come down to a risk responsibility transfer, from the public authorities to individuals and "communities", participating thus to the spread of a market rationality within the whole social life. However, few studies are dealing with the concrete effects of adaptation and resilience programs on the targeted populations.

From an ethnography of resilience and territorial adaptation to the flooding risk project set up by the World Bank and the Senegalese State in the Dakar suburbs, this article aims to analyse the responsibility transfers production linked to resilience operationalization. It shows that the configuration of power set up through this programme is more articulated to, than fully integrated to the neoliberal governmentality.

MOTS-CLÉS. Gouvernementalité, néolibéralisme, risque, inondations, résilience.

KEYWORDS. Governmentality, neoliberalism, risk, floods, resilience.

\section{Introduction}

Passant de la psychologie à l'écologie dans le courant des années 1970 la notion de résilience s'est imposée au cours des années 2000 comme l'une des figures centrales du traitement des risques et des catastrophes (Krieger, 2016). Très souple dans sa définition et permettant une réappropriation par de nombreuses disciplines, la notion est désormais largement mobilisée dans les politiques urbaines de tout ordre (Meerow, Newell, 2016, Meerow et al., 2016) et constitue un nouveau lieu commun des politiques internationales (Pugh, 2014).

Cette réappropriation par les institutions internationales a cependant fait récemment l'objet de nombreuses critiques, affirmant d'une part la porosité de la résilience à des principes néolibéraux tels que la "nécessaire » adaptation permanente de l'individu aux transformations de son environnement impliquant un rejet de l'état de stabilité et de sécurité (Welsh, 2012), d'autre part la dépolitisation de cet environnement au profit d'une « ontologie du changement » (Felli, 2014). La résilience procéderait 
ici d'une forme de "gouvernementalité » spécifique (Foucault, 1994 ; Joseph, 2013 a.), néolibérale, qui s'appliquerait dans des domaines aussi variés que la sécurité ou l'environnement. Dans ce modèle l'Etat et le marché définissent la norme et les prix de biens communs que les individus et communautés « responsables » doivent désormais « sécuriser » par eux-mêmes (Reid, 2012). La résilience y apparait comme un mode de gouvernement à distance, un «régime de subjectivation $»^{2}$, favorisant l'incorporation par les individus de techniques de soi et de manière de se penser en sujets responsables et adaptables dans un monde que l'Etat ou les autres institutions sociales stables (la classe sociale, l'identité etc.) n'ont plus le pouvoir, ou le souhait, de réguler (Evans, Reid, 2015, Biermann et al. 2015). Le succès de la notion dans les discours et orientations des grandes institutions internationales indique par ailleurs, pour de nombreux auteurs, un infléchissement profond des politiques transnationales. A partir des normes et discours portés par ces institutions, Romain Felli ajoute ainsi une quatrième « grande phase normative de la politique environnementale » aux trois autres identifiées par Bernstein (2001), laquelle serait « structurée autour de l'adaptation et promouvant une éthique de la résilience » (Felli, 2014). Chandler quant à lui, identifie dans les formes de gouvernement à distance promues par les politiques sécuritaires internationales et mettant l'emphase sur les notions d'empowerment et de résilience, un « shift » post-libéral (Chandler, 2012).

Ces transformations font cependant l'objet de débats importants parmi les tenants d'un positionnement critique. Jonathan Joseph (2013 a. et b., 2016) fait ainsi remarquer que, si tournant néolibéral il y a avec l'apparition et l'utilisation massive de ce concept ces 15 dernières années, son analyse doit séparer l'échelle étatique de l'échelle des populations pour être pertinente. L'accent mis sur la participation et «l'empowerment» de ces dernières face aux risques et aux catastrophes dans les politiques internationales appliquées aux "pays en développement» ne serait qu'un nouveau moyen pour les bailleurs et les agences des «pays développés » de justifier l'approfondissement des réformes libérales de l'Etat, véritables cibles des politiques de résilience (Joseph, 2016). De plus, l'approche en termes de gouvernementalité contribuerait à gommer la potentielle critique de la notion en aplanissant les négociations, conflits et réappropriations de la notion par les acteurs chargés de la mettre en œuvre dans les politiques internationales (Corry, 2014). Jessica Schmidt (2015) remarque pour sa part que la congruence entre néolibéralisme et résilience apparait un peu trop «intuitive», et que la lecture foucaldienne de sa réappropriation par les différentes institutions s'en revendiquant empêche d'en comprendre totalement les effets sur les modes de gouvernance et sur la pensée libérale. En tant qu'ensemble «d'opérations sur des champs de possibilités où vient s'inscrire le comportement de sujets agissants » (Foucault, 1994, p. 237), la notion de gouvernementalité impose ainsi de s'interroger sur l'opérationnalisation des concepts et leur champ d'application, ainsi que sur le régime de subjectivation en cause (Bayart, 2004). C'est en effet dans son application pratique que la résilience prend son sens politique (Djament-Tran et al. 2011). Dans la lignée de ces écrits insistant sur la nécessaire prise en compte des contextes et réappropriations locales dans l'analyse de la résilience, cet article vise à interroger l'hypothèse de la résilience comme outil d'extension d'une gouvernementalité néolibérale dans les villes des pays du Sud, à travers son application aux populations d'un territoire, la banlieue de Dakar, au Sénégal.

Depuis 2012 en effet, la Banque Mondiale et l'Etat sénégalais conduisent un programme de lutte contre les inondations affectant la banlieue de Dakar, le « Projet de gestion des eaux pluviales et d'adaptation au changement climatique » (PROGEP), devant servir de matrice à tous les programmes de mise hors d'eau de la zone. Saisir la résilience dans ce type de programmes n'est pas chose aisée, tant sa définition semble labile et son champ d'application large (Comfort et al., 2010). Même si le PROGEP et certains de ses acteurs mobilisent la notion de résilience ainsi qu'un champ sémantique relatif à l'adaptation des populations et du territoire face aux risques, sa mention varie fortement en fonction du type de documents en cause et des interlocuteurs, et elle n'est quasiment pas mobilisée sur le terrain. Elle nécessite donc d'être «située » dans des pratiques pour pouvoir être appréhendée, ce

\footnotetext{
${ }^{2}$ C'est ainsi que Bayart définit la gouvernementalité en citant Deleuze qui la perçoit comme « la production de modes d'existence ou de styles de vie » (Bayart, 2004, p. 196) (Deleuze, 1990, p. 156). 
que nous ferons après avoir retracé brièvement la genèse de ce programme et son mode d'action. En l'occurrence, c'est l'analyse des pratiques de «responsabilisation » qui sera privilégiée comme point commun, mis en lumière par la littérature, à la résilience et aux formes de gouvernementalité néolibérales, en vue d'interroger la pertinence de ce rapprochement. Nous tenterons par ce biais de comprendre les fondements pratiques et moraux des formes de délégation de responsabilité vis-à-vis du risque, des pouvoirs publics vers les populations. Puis nous verrons que cette responsabilisation des populations prend bien la forme d'un régime de subjectivation vis-à-vis du risque d'inondation, mais qu'il a pour effet de remettre l'Etat au centre du traitement des risques dans ces quartiers. Nous plaiderons finalement pour une meilleure appréhension des différentes logiques à l'œuvre dans les programmes se revendiquant d'une opérationnalisation de la résilience en vue de mieux situer le néolibéralisme dans son articulation avec d'autres régimes de pouvoir au sein des politiques internationales.

Méthode d'enquête et de recueil des matériaux

Cet article se base sur une recherche doctorale en sociologie portant sur les risques d'inondations dans la banlieue de Dakar. Les matériaux recueillis l'ont été au cours d'un « terrain ethnographique translocal » (Ullberg, 2013) de 13 mois, divisés en deux périodes de 6 et 7 mois entre mars et août 2015, puis entre mars et octobre 2016. Il s'est agi de suivre les acteurs et le risque d'inondation à travers une ethnographie de " plusieurs sites en même temps » (Ullberg, 2013, p. 213) et d'articuler des périodes de participation observante (Tedlock, 1991) intenses à des périodes d'observation participante plus resserrées dans le temps et l'espace en fonction des opportunités offertes par le terrain et du cheminement théorique et pratique du chercheur. Au sein des réflexions sur l'ethnographie multi-sites (Marcus, 1995), la perspective «translocal» nous permet d'insister sur les liens entre acteurs au sein d'une même ville et sur la dépendance du chercheur à ces liens pour construire son propre terrain ethnographique (Hannerz, 2003). Les matériaux présentés dans cet article sont issus plus spécifiquement d'observations étalées sur la deuxième période de terrain et réalisées aux côtés des acteurs des programmes dont il sera question, ainsi que sur une période de deux mois de présence quotidienne aux côtés de certains des acteurs de la lutte contre les inondations dans la commune de Djiddah Thiaroye Kao. Ces matériaux ont été complétés par 63 entretiens semis directifs menés auprès de différents acteurs des inondations (habitants, associations, opérateurs des programmes de l'Etat et de la banque mondiale, représentants d'ONG), pouvant durer d'une à trois heures, et réalisés, pour la plupart des entretiens avec des professionnel des programmes observés que je cite dans cet article, dans leurs bureaux respectifs. Je me suis aussi appuyé sur des documents de projets plus ou moins opérationnels (allant des documents de présentation aux bailleurs à certains dossier d'appel d'offre pour les travaux), sur des documents d'évaluation et de diagnostic, ainsi que sur des documents de communication, récoltés au fil de mon terrain et via des recherches sur internet (pour des exemples de ces documents voir note 12). Enfin, une partie des résultats présentés ici s'appuient sur une compilation d'articles de journaux parus dans «Le Soleil» (quotidien national, souvent présenté comme "pro-gouvernemental») sur les périodes s'étalant de juillet à septembre 2005 et de juillet à septembre 2009 (deux périodes d'importantes inondations).

\section{La résilience dans les programmes de lutte contre les inondations dans la banlieue de Dakar}

Depuis la toute fin des années 1980, l'agglomération dakaroise est régulièrement affectée par des inondations d'ampleur variable (Thiam, 2011). Touchant principalement les villes limitrophes à Dakar, Pikine et Guediwaye, ces inondations n'ont d'abord fait l'objet que de peu de considérations par l'Etat sénégalais, ce dernier se limitant à réagir aux catastrophes aux côtés d'un ensemble complexe et 
disparates d'ONG et d'acteurs humanitaires (Schaer et al., 2017). En 2005 cependant, de nombreuses communes ainsi que certains quartiers de la ville de Dakar elle-même subissent un épisode majeur d'inondations durant la saison des pluies, causant des dégâts humains et matériels très importants ${ }^{3}$, entrainant de fortes mobilisations populaires, et conduisant à l'annonce du premier plan étatique de prévention et de lutte contre les inondations ${ }^{4}$, le Plan Jaxaay. A l'époque, ce programme vise principalement à déplacer les habitants des quartiers considérés comme inondables vers de nouveaux logements construits par l'Etat et situés en grande périphérie de l'agglomération. Le programme accumule cependant au fil des années un retard considérable, est entaché de scandales de corruption, et ne parvient pas à prévenir de nouveaux épisodes majeurs d'inondations comme ceux de 2009 et 2012.

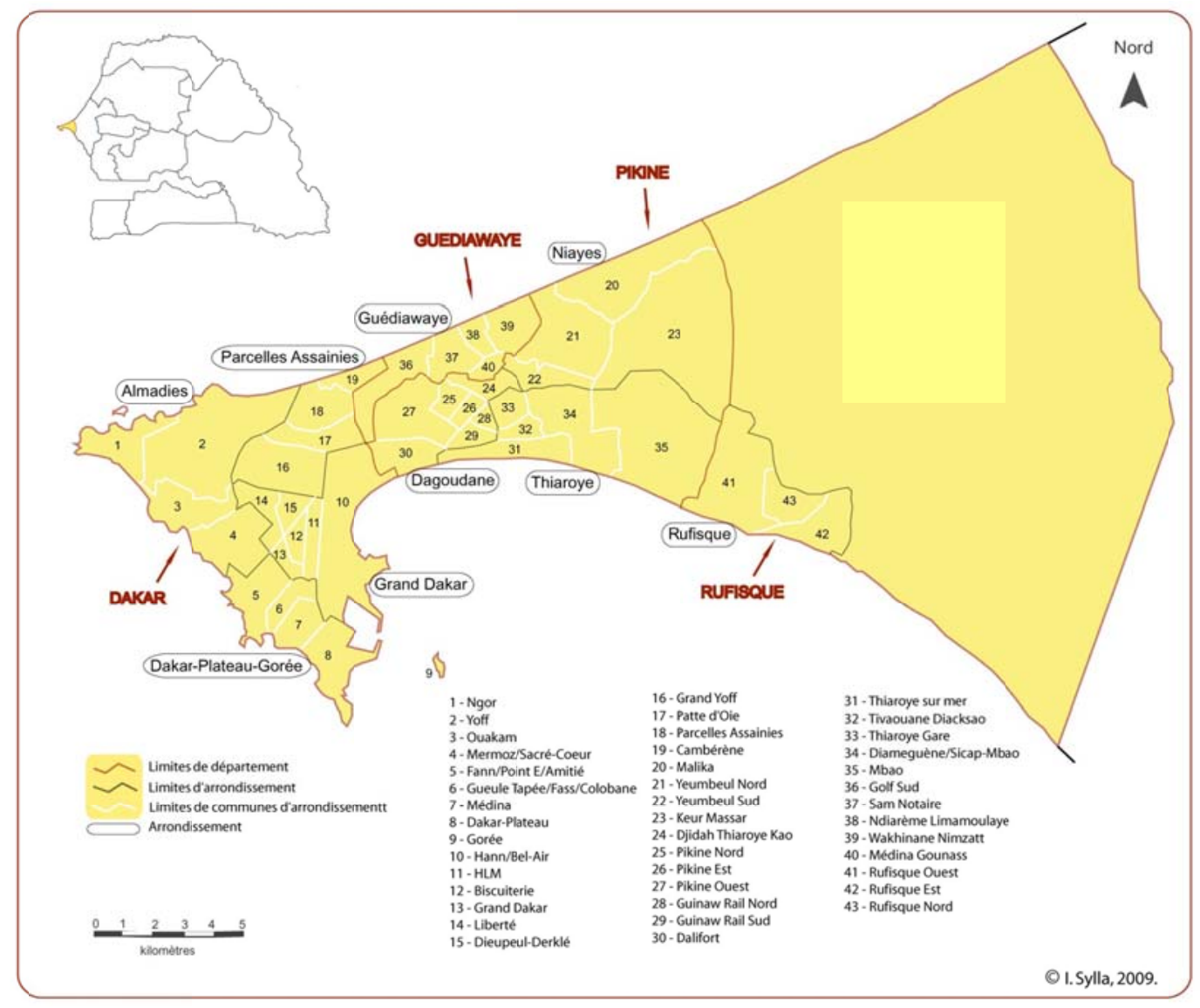

Carte 1. Carte de situation des différentes Villes et communes de l'agglomération dakaroise (Sylla, 2009)

Officiellement lancé en $2012^{5}$, le Projet d'adaptation au changement climatique et de gestion des eaux pluviales (PROGEP) fait suite au «rapport post catastrophe» de 2009 produit par la Banque Mondiale, qui chiffre pour la première fois les dégâts causés par les inondations. Il est conduit par l'Agence de Développement Municipale (ADM), une agence chargée d'accompagner les communes

\footnotetext{
${ }^{3}$ Nous n'avons pas retrouvé d'évaluation exacte des dommages causés par les inondations de 2005 mais les journaux de l'époque signalent une résurgence des maladies hydriques comme le choléra ainsi qu'une explosion du paludisme dans les quartiers affectés. L'impact des inondations sur l'économie, largement informelle, de ces quartiers est aussi souligné et les vastes zones de maisons abandonnées indiquent l'importance des dommages causés par les inondations. Par comparaison, les inondations de 2009 considérées comme relativement similaires à celles de 2005, ont causé 56 millions de dollars de dommages et 48 millions de pertes malgré le fait qu'à l'époque, une large part des zones très inondables étaient déjà abandonnées et sans compter les investissements préalables réalisés par les habitants pour protéger leur maison des eaux (remblais, surélévation dies murs etc.), (Cissé, Sèye, 2015). Pour un rapport complet sur l'impact des inondations de 2009 voir le rapport de la Banque mondial : World Bank report: World Bank, Senegalese State, 2010. Rapport d'évaluation des besoins post catastrophe. Inondations urbaines à Dakar 2009.

${ }^{4}$ Avant cette date, l'action de l'Etat passe principalement par la mobilisation de fonds d'assistance et de secours aux sinistrés (à l'instar de nombreuses ONG). Cette réponse à la catastrophe apparait ainsi comme réactive plus que préventive. Le plan Jaxaay rompt ainsi nettement avec cette logique (Thiam, 2011).

${ }^{5}$ Les premières discussions et actions autour du PROGEP datent en fait de 2009 et surtout 2010. Elles associent la Banque mondiale et l'Agence de Développement Municipale (ADM) dont l'action consiste à articuler les collectivités locales décentralisée et les bailleurs de fonds. L'ADM est largement liée à l'Etat, par sa structure organisationnelle comme par la composition de son personnel. (C) 2017 ISTE OpenScience - Published by ISTE Ltd. London, UK - openscience.fr 
dans leurs efforts de développement et de gestion urbaine, fonctionnant en grande partie sur des fonds issus des institutions international et sur un budget alloué par 1'Etat ${ }^{6}$. Il couvre par ailleurs principalement les Villes de Pikine et de Guediawaye pour ce qui est de la planification et d'une partie de la réalisation des ouvrages physiques. Ces derniers se concentrent pour les deux premières phases du projet principalement dans les communes de Dalifort, Djidah Thiaroye Kao, Wakhinane Nimzatt, Médina Gounass, Yeumbeul Sud et Yeumbeul Nord ${ }^{7}$. Comme son nom l'indique, le PROGEP vise à « adapter» le territoire aux changements climatiques mais aussi à "préserver les populations vivant dans les zones sujettes aux inondations [et à] promouvoir la gestion axée sur la durabilité et la résilience [...] par le biais de renforcement des capacités des acteurs de la gestion urbaine $»^{8}$. Il se situe ainsi dans le cadre du «Dispositif mondial pour la réduction des effets des catastrophes et le relèvement » (GFDRR) dont l'objectif est de « mettre au centre » du traitement des catastrophes et des risques la notion de résilience ${ }^{9}$. Le PROGEP a en outre pour vocation d'influer sur les futurs projets de mitigation des inondations dans la banlieue de Dakar et il est à ce titre présenté comme le principal outil des politiques sénégalaises de lutte contre les inondations ${ }^{10}$. Le volet « mise hors d'eau » du projet Pikine Irrégulier Sud (PIS) ${ }^{11}$, financé là encore par l'Etat sénégalais et la Banque mondiale, et complété en 2015 par l'Agence Française de Développement (AFD), suit ainsi le Plan directeur de drainage produit par le PROGEP et applique très largement ses principes directeurs.

La notion de résilience apparait cependant assez peu mobilisée par les opérateurs de ces projets et encore moins par les opérateurs « de terrain $»^{12}$ et par les populations censées bénéficier de ces actions. Reproduisant cette dernière distinction entre opérateurs, le champ sémantique de la résilience apparait globalement absent des documents de suivi, des rapports sur l'état d'avancement et des études sectorielles tandis que les documents d'orientation que sont les études de diagnostic et de préconisation, les documents de projet en détaillant la logique générale, et certains documents de communication l'utilisent largement ${ }^{13}$. A ce niveau donc, l'action portée par le PROGEP apparait

\footnotetext{
${ }^{6}$ A Banque mondiale a investi 70 millions de dollars dans ce projet tandis que l'Etat lui-même contribue à une hauteur qui m'est inconnue mais sans doute conséquente puisqu'ils indemnisent les ménages déplacés sur fonds propre.

${ }^{7}$ Depuis I'acte III de la décentralisation de 2013, l'agglomération de Dakar est découpe en 4 « Villes » (Dakar,Guediawaye,Pikine et Rufisque) eux-mêmes composés de communes d'arrondissements (voir carte 1), Sané, 2016.

${ }^{8}$ Agence de Développement Municipale, PROGEP, financements additionnels, cadre de gestion environnemental et social actualisé, approuvé et publié en Décembre 2011, actualisée en Janvier 2015.

${ }^{9}$ Voir "Gérer les risques des catastrophes pour protéger le développement ", Site de la Banque Mondiale, 11 avril 2014, url: http://www.banquemondiale.org/fr/results/2013/04/12/managing-disaster-risks-resilient-development [consulté le 25/04/2017]

${ }^{10}$ Suite à son inscription dans le programmes 100 resilient cities lancé en 2013 par la fondation Rockefeller, la ville de Dakar s'est dotée en décembre 2016 d’un document présentant la " stratégie de résilience » de la ville (Ville de Dakar, 2016. " Stratégie de résilience de la Ville de Dakar ", document en ligne, URL: http://www.100resilientcities.org/wpcontent/uploads/2017/07/Dakar_Resilience_Strategy_FRANCI\%CC\%80\%C2\%A7AIS.pdf). Ce dernier ne concerne que la Ville de Dakar et n'inclue donc pas les Villes Pikine et de Guediawaye qui rassemblent les principales communes de la banlieue et qui constituent les principales cibles du PROGEP. La confusion entre ces différentes entités est cependant entretenue jusque dans ce dernier document puisque les chiffres qui y sont avancés pour décrire le terrain d'intervention du programme concernent tour à tour la Ville de Dakar uniquement, et l'ensemble de l'agglomération, sans que la distinction soit clairement établie.

${ }^{11}$ Pikine Irrégulier Sud est en fait une partie de la banlieue de Dakar qui rassemble les 9 communes traversées par la nouvelle autoroute à péage reliant Dakar centre à Diamniadio. La construction de l'autoroute a conduit au déplacement massif des populations de ces quartiers précaires. Le projet Pikine Irrégulier Sud et principalement son volet " mise hors d'eau » fait suite à la construction de l'autoroute et intervient en complément, si ce n'est en compensation, de la traversée de ces quartiers par cette autoroute.

12 J'utilise cette expression, "opérateur de terrain ", pour désigner les personnels de ces projets chargés de leur mise en œuvre " technique » et n'ayant pas de prises sur les orientations générales et stratégiques de ces projets, même si un même individu peut plus ou moins cumuler ces deux fonctions.

${ }^{13}$ Voir par exemple respectivement: World Bank, GFDRR, UNDP, UE, Etat du Sénégal, 2014, " Sénégal : Inondations urbaines. Le relèvement et la reconstruction à partir de 2009 ", Série d'étude de cas, URL: https://www.gfdrr.org/sites/gfdrr/files/Senegal French Sept\%202014.pdf; World Bank, 2010, "Project document. Concept stage ", URL :
}

http://documents.banquemondiale.org/curated/fr/729201468306836716/pdf/PIDOFloodOproject0Concept0Stage0Nov012.pdf ; Agence de Développement Municipale (2011, actualisé en 2015), PROGEP, financements additionnels, cadre de gestion environnemental et social actualisé. ;

World Bank, GFDRR, "Stories of impact. Making Senegal's cities more flood resilient ", URL http://documents.worldbank.org/curated/pt/334961495610181592/pdf/115241-BRI-PUBLIC-ADD-SERIES-soi-senegal-flood.pdf (c) 2017 ISTE OpenScience - Published by ISTE Ltd. London, UK - openscience.fr 
structurée autour de trois principaux volets visant d'une part à inclure les risques d'inondations dans la gestion urbaine, d'autre part à adapter le territoire à ces risques, enfin à inclure les populations et « communautés » dans leurs préventions et à favoriser leur résilience ${ }^{14}$. C'est dans ce dernier volet que la notion de résilience va être la plus mobilisée, sans être définie pour autant, et bien que la notion et son champ sémantique soient aussi utilisés pour décrire la logique générale du projet ${ }^{15}$. L'ensemble de ces volets s'inscrivent ainsi dans une narration ${ }^{16}$ faisant de la résilience urbaine (par le biais de « l'adaptation du territoire ») un objectif à atteindre par la planification urbaine et la clarification du schéma institutionnel de gestion du risque, ainsi que par la mobilisation et l'éducation des populations et « des communautés touchées afin de promouvoir un changement de comportement et une résilience face aux risques $\rangle^{17}$. La mobilisation du concept de narration nous permet ici d'insister sur la cohérence du raisonnement dans ce projet, mais pas sur son caractère hégémonique. La narration de la résilience y côtoie en effet des rationalités plus «classiques» dans le champ du développement, propres à la planification, à la nécessaire implication des communautés etc. En ce sens, l'analyse des projets se réclamant de la résilience, mais mobilisant aussi d'autres perspectives parfois en contradiction les unes avec les autres, est forcément une «lecture » de ces projets sous cet angle par le chercheur. Le champ lexical mobilisé dans les documents de projet et l'orientation générale visant non pas à empêcher la catastrophe mais à s'y adapter, à l'échelle urbaine comme à celle des individus et des " communautés », de même que son ancrage institutionnel et la communication l'entourant, situe ainsi le PROGEP dans la perspective de la résilience comme nouvel opérateur des politiques internationales environnementales telles qu'analysées par les auteurs y voyant une nouvelle forme prise par la « gouvernementalité néolibérale».

Le concept apparait cependant articulé à d'autres logiques et peu opérationnel alors même que la résilience ne devient une éthique individuelle (et donc une forme possible de «technique de soi ») dans le discours des institutions internationales qu'à partir du moment où elle est conceptualisée comme une qualité qu'il est possible de produire (Felli, 2014). De même, c'est bien dans son opérationnalisation dans certaines politiques publiques que la résilience intègre une forme de gouvernementalité sous la plume de Jonathan Joseph (2013 a.). Il apparait donc nécessaire de s’intéresser à la manière dont les opérateurs de ces projets de résilience en spécifient la logique générale dans leur action.

\section{Des populations responsables ou coupables? Situer la résilience dans les pratiques des acteurs}

En pratique en effet, la résilience apparait difficile à situer en dehors des documents de projet. Les opérateurs des programmes eux-mêmes y font peu référence, sinon comme un cadre à «l'implication des populations » dans ces programmes (nous le verrons). Leur discours apparait en revanche saturé de formes variables d'attribution de responsabilités, par rapport aux inondations ou dans la conduite de l'action. Nécessaire à la conduite de toute action concertée, la responsabilisation des acteurs prend un sens particulier dans le cadre de l'opérationnalisation de la résilience. Comme évoqué en introduction, la principale critique adressée à cette notion dans son application par les politiques internationales apparait dans le transfert de la responsabilité d'anticipation et de protection vis-à-vis du risque, qui passerait de l'Etat vers les communautés et individus, contribuant ainsi à dépolitiser et à individualiser cette question (Reid, 2012; Djament-Tran et al. 2011). C'est principalement dans ce déplacement de responsabilité que s'opère le rapprochement avec la gouvernementalité néolibérale (Joseph, 2013 a.), dans un contexte où l'autogouvernement des individus constitue l'un des points saillants de l'analyse

\footnotetext{
${ }^{14}$ Un quatrième volet est relatif à la coordination, à la gestion et au suivi du projet mais nous intéresse moins dans le cadre de cet article.

${ }^{15}$ Une chargée de projet de la Banque Mondiale décrira par exemple l'ensemble de l'action comme de la « résilience urbaine » et de nombreux documents de communication utilisent ce terme.

${ }^{16}$ Pour une discussion des manières dont la résilience est perçue et conceptualisée en tant que narration par la recherche et dans les politiques publiques, voir Béné et al., 2017.

${ }^{17}$ Agence de Développement Municipale, PROGEP, financements additionnels, cadre de gestion environnemental et social actualisé, approuvé et publié en Décembre 2011, actualisée en Janvier 2015. 
des formes d'indirect rules portées par le néolibéralisme (Hache, 2007 a. et b. ; Fournier, 2015). Cette catégorie nous est apparue d'autant plus pertinente pour appréhender des pratiques d'opérationnalisation de la résilience qu'elle fonde largement l'action du PROGEP dans la banlieue de Dakar.

Comme la plupart des actions concertées relatives à l'identification et au traitement d'un risque, le PROGEP se construit sur une narration, cette fois propre au projet lui-même, organisant les menaces et identifiant de manière partielle et sélective des causes ou des séries d'événements ayant conduit, ou susceptibles de conduire à la catastrophe. Objet de controverse entre différents acteurs de la lutte contre les inondations, ce récit est une production sociale à part entière, située politiquement, qui conditionne largement le type de solutions mises en place par le PROGEP ${ }^{18}$. Aujourd'hui partagé par de nombreux journalistes, par l'ensemble des opérateurs des programmes dont il est question dans cet article et par une partie des habitants des quartiers inondés, ce récit peut se résumer comme suit ${ }^{19}$ :

Dans les années 1970 et 1980, une forte sécheresse touche le Sénégal et la région de Dakar, asséchant partiellement le réseau de Niayes, c'est-à-dire le réseau hydrographique «naturel » de la région. A cette période, de nombreux «migrants de l'intérieur », poussés par la sécheresse, viennent s'installer dans la région de Dakar où ils bénéficient de l'achat informel (en dehors de la législation réglementant l'accès aux terres et en dehors de toute planification urbaine) de parcelles dans des zones autrefois humides. Avec le retour des pluies à la fin des années 1980, les eaux « reprennent leur lit naturel » au sein des quartiers et des maisons nouvellement construites, causant de fait des inondations. En conséquence, dans ce récit, les actions ponctuelles de pompage menées par les collectifs d'habitants, les autorités publiques et certaines agences gouvernementales en vue de secourir les quartiers inondés ne résolvent pas le problème structurel qui est avant tout d'ordre topographique. L'action du PROGEP et des programmes publics de «mise hors d'eau » de ces quartiers va donc consister dans la délimitation des zones inondables rendues inconstructibles et dans la recréation de ces anciennes voies d'eau naturelles en vue d'évacuer les eaux de pluie de l'intérieur des quartiers vers la mer selon une « méthode de drainage gravitaire ${ }^{20}$.

Ce récit nous apparait remplir deux fonctions dans la conduite de l'action dans les programmes d'adaptation du territoire de la banlieue de Dakar au risque d'inondation. La première est d'ordre moral et consiste en l'attribution d'une responsabilité aux habitants des quartiers précaires ${ }^{21}$ dans les inondations dont ils souffrent. La cause première des inondations est en effet localisée, dans ce récit, au tournant des années 1970, lorsque des migrants sans toits deviennent des habitants sans droits en s'installant dans des zones inondables en dehors de la législation. La responsabilité ainsi attribuée prend la forme d'une faute et justifie le traitement du risque par le déplacement des populations dans des zones périphériques. Déjà à la base de la mise en œuvre du plan Jaxaay en 2005, cette fonction s'articule à une autre d'ordre pratique dont nos entretiens laissent à penser qu'elle se construit en partie entre le début de ce dernier programme et le rapport post-catastrophe de la Banque mondiale, à mesure que certains acteurs du traitement du risque, hydrologues principalement, montent dans l'appareil d'Etat et rencontrent les experts de la Banque. En spécifiant la nature du problème, hydrologique et topographique, ce récit induit une approche technique du risque ainsi qu'une échelle d'intervention, celle des bassins versants et donc de la région plutôt que celle des villes et des communautés. Il permet

\footnotetext{
${ }^{18}$ Sur la construction de ces narrations, les controverses auxquelles elles ont donné lieu dans la banlieue de Dakar, et sur leur importance dans la conduite même de l'action contre le risque, voir Leclercq, 2017.

${ }^{19}$ Ce résumé est une reconstruction de l'auteur basée sur les entretiens menés avec les opérateurs du PROGEP, de PIS, certains habitants et acteurs municipaux de la commune de Djiddah Thiaroye Kao (Pikine) et sur le dépouillement d'une partie de la presse sénégalaise sur le sujet. Pour plus de détail sur ce récit, voir Leclercq 2017.

${ }^{20}$ Il s'agit en fait de construire des canaux de drainage ainsi que des bassins de rétention d'eau selon la topographie en vue de permettre l'écoulement des eaux jusqu'à la mer sans avoir à les pomper.

${ }^{21}$ Le terme "quartiers précaires " désigne ici des quartiers constitués pour une part importante de logements construits en dehors de la législation réglementant l'accès à la terre et la construction, aussi appelé " logements informels ». Dans la banlieue de Dakar, ces logements sont souvent construits "en dur », et existent depuis parfois plus de 50 ans. Pour une revue de la question particulièrement complète, voir Deboulet, 2016. 
aussi de légitimer l'action du PROGEP par rapport à celle d'autres acteurs présents sur ce terrain. Sous l'angle qui nous intéresse, il rend les experts détenteurs de la «bonne » approche, responsables de l'adaptation du territoire aux inondations ${ }^{22}$.

Partant de notre postulat que l'emploi de la résilience comme concept opératoire produit des effets observables sur le traitement du risque, il nous apparait que les formes de responsabilisation peuvent donc être saisies comme des pratiques susceptibles de rendre compte de cette opérationnalisation et d'une possible congruence avec des formes de gouvernementalité néolibérale. Si les transferts de responsabilité ne sont pas propres à la résilience, ils permettent en effet aux acteurs des programmes s'en revendiquant ici de justifier leur approche, tout en fournissant un cadre au chercheur pour penser les formes politiques des pouvoirs à l'œuvre. Ils nécessitent cependant d'être observés dans leurs applications quotidiennes au sein de ces programmes et auprès des « populations cibles ».

\section{La résilience comme mobilisation « communautaire » autour des projets de l’Etat}

Sur le terrain des pratiques, le récit évoqué ci-dessus se traduit par un dispositif complexe dans lequel des hydrologues de l'ADM et des bureaux d'études mobilisés établissent le tracé des anciennes voies d'eau « naturelles » à « recréer » grâce à des logiciels de gestion des flux et à une modélisation numérique de la topographie de la région à traiter. Ils construisent ainsi un réseau théorique de canaux de drainage et de bassins de rétention des eaux (voir figure 1). En fonction du degré d'avancement des programmes $^{23}$ et la délimitation définitive du périmètre des ouvrages, le tracé de ces canaux et de ces bassins est matérialisé et délimité dans les quartiers par des géomètres. Le délégué du préfet ${ }^{24} \mathrm{se}^{\mathrm{s}}$ charge alors d'établir la liste des habitations ou équipements impactés et la formalise dans un plan ad hoc. Ces habitants impactés sont informés par des médiateurs sociaux ${ }^{25}$ qui vont alors les aider à rassembler les documents nécessaires à l'indemnisation accordée par l'Etat en compensation des habitations détruites ${ }^{26}$. Cette indemnisation se négociera, sur la base d'une recension des biens partiellement ou totalement détruits et de la valeur du foncier réquisitionné, dans une commission de conciliation à la préfecture de Pikine. Les ménages affectés par le projet pourront alors être relogés dans les différentes zones prévues par l'Etat et la Banque Mondiale en grande périphérie de l'agglomération (les cités Jaxaay et Tawfeex, voir figure 2) ou garder l'ensemble de l'indemnité et chercher à se reloger par leurs propres moyens. Des formes de négociations (du périmètre des ouvrages, de leur tracé...) existent tout au long de ce processus mais elles n'impliquent pas les populations (ou à la marge, lorsqu'un terrain impacté par le projet et revendiqué par son « propriétaire » informel alors qu'il n'avait pas été recensé). En définitive, les populations n'ont de marge de manœuvre qu'au moment de la négociation des formes et du montant de leur indemnisation $^{27}$. Les ménages habitant dans les périmètres de construction des ouvrages de drainage

\footnotetext{
${ }^{22}$ Ce récit en côtoie un autre portant sur la responsabilité de l'Etat, et d'autres acteurs telles que les ONG ou l'aide internationale dans la gestion disparate et sporadique du problème avant 2010. De fait, l'un des volets du PROGEP que nous n'abordons pas dans cet article concerne l'organisation des différentes institutions dans la gestion du territoire et de la lutte contre les inondations, et l'un des apports principaux du PROGEP consiste dans la formalisation d'outils de gouvernance comme le Plan Directeur de Drainage. Pour plus d'informations sur ces aspects, voir Schaer et al., 2017 et Leclercq, 2017.

${ }^{23} \mathrm{~J}$ 'inclus dans ces programmes le PROGEP mais aussi le volet " mise hors d'eau » du programme PIS évoqué précédemment.

${ }^{24}$ La gestion des expropriations, des indemnisations et des plans de réinstallation des populations est à la charge de la préfecture sous l'autorité du ministère de l'intérieur.

${ }^{25}$ Ce travail de médiation sociale est attribuée au cours du projet à une « ONG d'appui » ou à un bureau d'étude spécialisé.

${ }^{26}$ Cette indemnisation résulte de la politique opérationnelle (OP 4.12) de la Banque Mondiale concernant la réinstallation involontaire de personnes et qui stipule que les personnes dont l'habitat ou le lieu de travail est impactés par les projets soutenus par la Banque Mondiale doivent être indemnisés pour la perte de leur habitat, de leurs biens ou accès aux biens, de leurs source de revenus ou moyens d'existence (Banque Mondiale, 2001, Manuel opérationnel de la Banque mondiale, Politiques opérationnelles, disponible en ligne : http://siteresources.worldbank.org/OPSMANUAL/Resources/OP412-French.pdf).

${ }^{27}$ Ces négociations ne portent pas alors sur le montant de chaque bien, fixé à l'avance par un barème en fonction du statut du terrain impacté (habitat irrégulier, habitat régulier, espace de travail, de vente, de culture etc.), du nombre et du statut de pièces détruites (chambre, cuisine...), du statut des occupants (locataires, propriétaires...) etc., mais sur le statut de chaque bien, leur nombre, ou sur les stratégies à adopter qui peuvent être directement discutées avec certains des représentants des ONG d'appui. II (C) 2017 ISTE OpenScience - Published by ISTE Ltd. London, UK - openscience.fr 
sont ainsi sélectionnées par les experts. Seuls ceux qui n’y sont pas inclus vont donc bénéficier des opérations de « renforcement des capacités des acteurs communautaires pour la gestion urbaine » dans les secteurs ciblés par le projet.

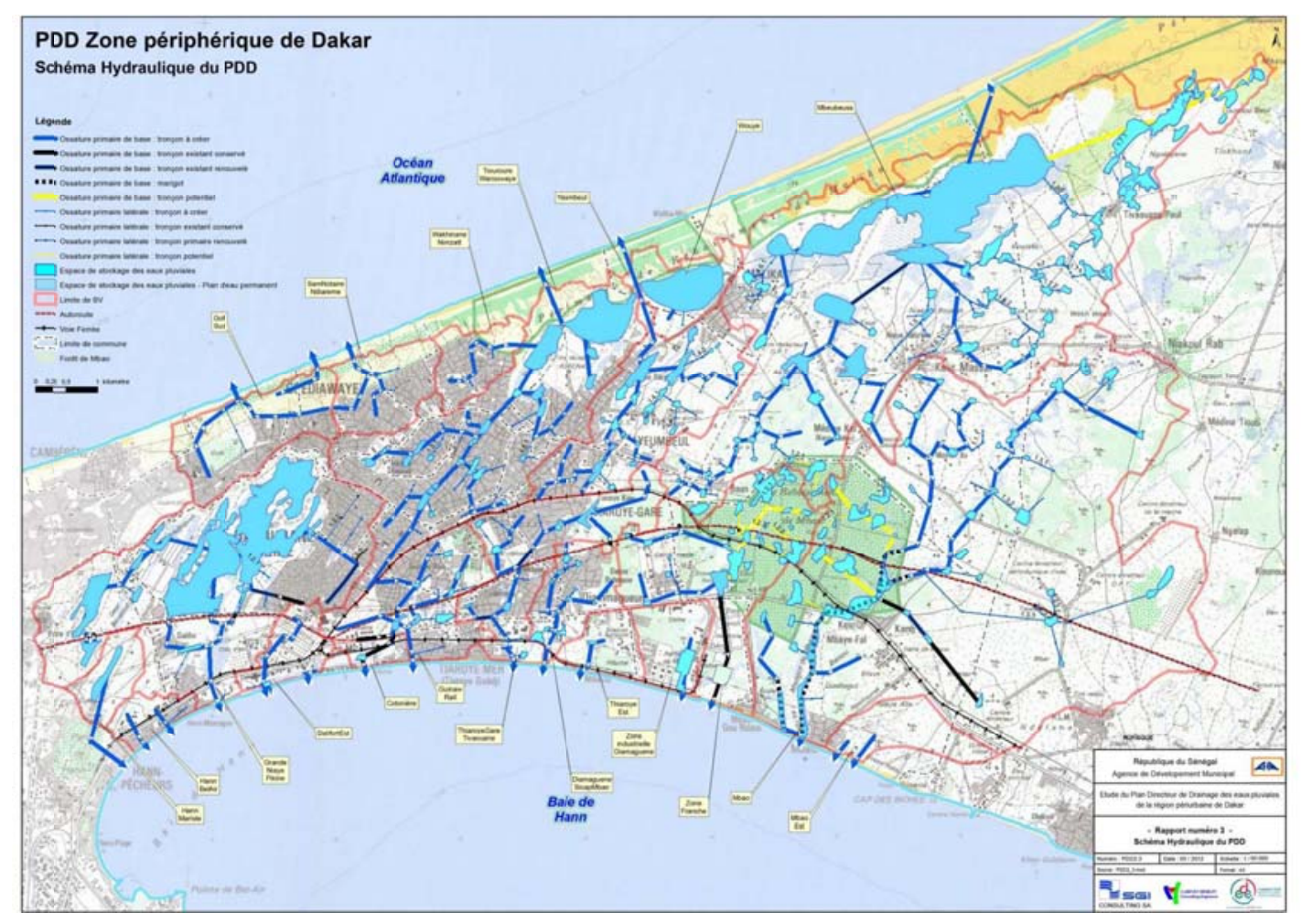

Figure 1. Plan Directeur de Drainage (PROGEP) : Les tracés bleus figurent les ouvrages de drainages primaires projetés ou déjà réalisés, tandis que les étendues bleues claires figures les lacs ou les bassins de rétention d'eau 


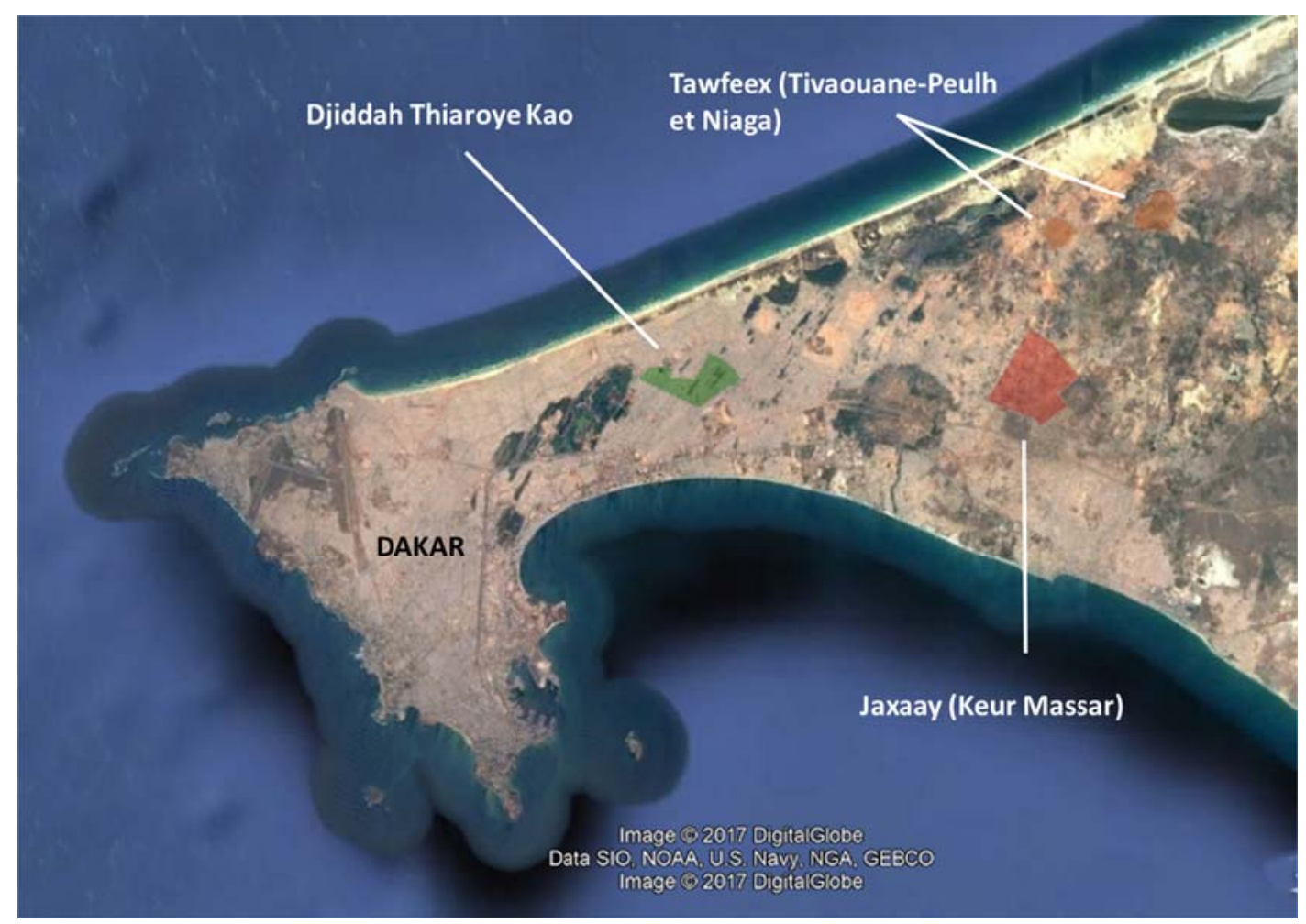

Figure 2. Emplacement des différents sites de logements sociaux mis en place par l'Etat sénégalais (Jaxaay et Tawfeex) et du terrain d'étude principal, la commune de Djiddah Thiaroye Kao

Largement basées sur la construction de dispositifs d'organisation et de coordination des « communautés » entre elles, avec les autorités et avec les équipes du PROGEP, ou d'actions directes de sensibilisation dirigées vers les habitants des quartiers à risque, les formes de responsabilisation des populations apparaissent au cœur de la stratégie de résilience communautaire de ce programme d'adaptation. Cependant, assez loin d'un outillage censé permettre la «prise en charge » du risque par les habitants eux même (Reid, 2012), la responsabilisation communautaire vis-à-vis du risque portée par ce programme et ses héritiers apparait principalement dirigée vers l'entretien des ouvrages de drainage évoqués précédemment. Des comités d'action locaux, présidés par le maire mais rassemblant des acteurs de divers collectifs, associations ou groupements, aux projets d'investissement communautaire concernant principalement les groupes de jeunes habitants aux alentours des bassins de rétention des eaux, tous ont comme principal objectif soit de surveiller la propreté des canaux et des bassins de rétention (et de faire « remonter les informations » en cas de problèmes), soit d'organiser le curage de ces derniers ${ }^{28}$, soit de mettre au point un projet d'aménagement des abords des bassins en vue de les valoriser et d'y rendre plus difficile le jet des ordures ${ }^{29}$. De même, le fort investissement des radios locales dites « radios communautaires », les actions auprès des collectifs déjà structurés comme les groupes d'épargne ou les associations de jeunes, ou les opérations directes de sensibilisation auprès des «notables » et des « leaders d'opinion » visent pour la plupart à prévenir les accidents lors des travaux et à réduire l'encombrement des bassins et canaux par les déchets. C'est que, comme l'explique un responsable du volet «participation communautaire » pour le PROGEP lors d'un entretien, « l'insalubrité » des quartiers est perçue à la fois comme une cause des inondations et comme un facteur susceptible d'entraver le bon fonctionnement des infrastructures de drainage.

\footnotetext{
${ }^{28}$ Par des opérations de set setal soit des opérations souvent ponctuelles de nettoiement d'un ou de plusieurs quartiers, menées par des groupes de jeunes. Ces opérations sont d'autant plus faciles à mettre en place qu'elles sont devenues depuis la fin des années 1980 une des modalités classiques d'engagement de ces groupes pour leur quartier, mais aussi plus largement une modalité d'engagement politique (cf. Fredericks, 2013).

${ }^{29}$ De fait, à Djiddah Thiaroye Kao, les abords du bassin de Bagdad par exemple sont aujourd'hui assez investis le soir comme lieu de rassemblement ou de sport ce qui limite le jet d'ordures ou d'eaux usées à cette période de la journée. 
«L'engagement communautaire, c'est lié au fait que d'abord, si on veut réduire les risques d'inondation, c'est par rapport à la population. Donc véritablement on revient à... au point nodal de l'intervention du PROGEP. D'une part les populations comme cause liée au risque d'inondation, mais d'autre part les populations comme bénéficiaires effectivement de la réduction des risques d'inondation (approbation de l'enquêteur). Et c'est de là effectivement qu'est née la composante $C^{30}$ qui porte beaucoup plus aujourd'hui sur l'engagement communautaire. Elle concerne le niveau d'engagement que la communauté doit avoir, la conscience collective qui doit être effectivement impulsée au sein de la communauté pour qu'une responsabilité collégiale puisse être portée par la communauté pour réduire les risques d'inondations. [...] Et... ces quelques choses aussi sont liées véritablement, faudrait-il le dire, à la problématique même de gestion des ouvrages de drainage, dans nos pays, par le fait que, on sait que naturellement les risques d'inondations ont une forte corrélation avec les phénomènes d'insalubrité.

\section{Enquêteur : C'est à dire ? En quoi ?}

En quoi ? Disons... aujourd'hui, quasiment, si on constate que les voix d'eau naturelles sont la plupart du temps obstruées par des ordures, de toutes natures, que ce soit des débris de construction, que ce soit des ordures ménagères parce qu'aussi ces sites sont souvent transformés en dépotoirs sauvages, que ce soit aussi les eaux usées. Donc, partant, le phénomène d'insalubrité participe effectivement à l'obstruction des voix d'eau naturelles. Donc ce qui nous fait dire que la problématique de gestion des eaux pluviales a une forte corrélation avec le phénomène d'insalubrité dans nos quartiers. [...] Maintenant parallèlement à ça aussi, dans une dynamique globale, pour préserver ces ouvrages par exemple de ces ordures, il faudrait que le maire puisse vraiment être dans les dispositions d'assurer vraiment un bon service de ramassage d'ordures pour ces populations. Aujourd'hui un problème qui se pose c'est plus par rapport aux eaux usées, il faudrait que le maire soit plus dans une dynamique de gestion des eaux usées avec la réalisation de puisards. »

(Alioune $^{31}$, Responsable Participation communautaire, 23/06/2016)

Si la responsabilisation des populations et «communautés » a clairement ici pour but d'entretenir les ouvrages de l'Etat plutôt que de «renforcer des stratégies endogènes » de lutte contre le risque, c'est la logique générale de ces actions qu'il s'agit de relever. La « culpabilité » des habitants dans les inondations est rappelée et articulée à la responsabilisation de ces mêmes habitants dans l'entretien des ouvrages de drainage. Cette responsabilisation apparait comme une forme de compensation nécessaire des habitants victimes/coupables en contrepartie de la construction de ces ouvrages. Les causes structurelles de ces dépotoirs sauvages qui obstruent le passage des eaux, dans les canaux de drainage et ailleurs, liées à la quasi inexistence de réseaux d'évacuation des eaux usées et à la faiblesse des systèmes d'évacuation des déchets solides dans ces quartiers, n'est évoquée que de manière détournée et naturalisée («dans nos pays »). La corrélation est donc établie entre « insalubrité », obstruction des voix d'eau et des ouvrages de drainage, et inondations, mais pas entre absence de réseaux d'assainissement et inondations, déclinant ainsi la narration des inondations sur laquelle se base le PROGEP en schéma opérationnel. Reconnue plus tard dans l'entretien, l'absence de ces réseaux d'assainissement et de collecte de déchets est considérée comme un problème de gouvernance « parallèle » à celui de l'entretien des ouvrages. Cet aspect est d'autant plus difficilement intégrable dans la logique globale présentée par ce responsable que le PROGEP ne porte pas d'action sur ces aspects, si ce n'est d'offrir un « cadre de discussion » pour permettre la rencontre entre les acteurs de la gestion des ordures, les maires etc.

\footnotetext{
${ }^{30}$ Il s'agit du volet « participation communautaire dans le drainage » du PROGEP, qui porte l'ensemble des actions de résilience au niveau des « communautés ".

${ }^{31}$ Les noms des personnes citées ont été modifiés.

(C) 2017 ISTE OpenScience - Published by ISTE Ltd. London, UK - openscience.fr 
Il ne s'agit pas ici de dénigrer l'action de ces acteurs qui supportent des programmes déjà très lourds financièrement et en termes de ressources humaines et techniques, mais de repérer les logiques principales de ces programmes, triant nécessairement entre les aspects plus ou moins urgents à traiter en fonction des possibilités et des budgets alloués, selon les orientations des bailleurs etc. Ce discours n'est en effet pas hors sol mais s'ancre dans des réalités sociales, politiques et urbaines avec lesquelles les concepteurs des projets aussi bien que leurs opérateurs sont obligés de composer. L'absence de réseaux d'assainissement en est une, aussi bien que les fonds limités en quantité et dans le temps pour l'adaptation du territoire aux inondations et la difficulté à trouver une institution pérenne et assez solide pour assurer, sur le temps long, l'entretien des ouvrages de drainage. Reste que la logique générale du projet, triant entre ces différents aspects, trouve dans le discours de la résilience et de l'adaptation basé sur la responsabilisation des individus et communautés par rapport au risque une justification dans le fait de ne pas prendre en compte ces problèmes structurels dans l'action entreprise sur le territoire (Joseph, 2016). En outre, si les acteurs de terrain que sont les comités ad hoc mis en place par le PROGEP ou les « facilitateurs sociaux » chargés de la sensibilisation des populations, dont certains sont issus directement des mouvements locaux de lutte contre les inondations, ne mobilisent pas la notion, ils promeuvent néanmoins le «récit» porté par ce dernier et les formes de responsabilisation associées ${ }^{33}$.

A la responsabilisation morale des habitants des quartiers précaires évoquée en première partie s'articule donc une responsabilisation pratique dans l'entretien des ouvrages érigés par l'Etat et la Banque mondiale. Cette dernière s'applique à des «communautés » expurgées des victimes directes des inondations et des projets d'adaptation selon une vision technique du territoire. Il y a bien différentes formes de rationalités qui s'articulent autour d'une action forte de l'Etat visant non pas uniquement à orienter et contraindre les populations et les flux mais aussi à modeler des dispositifs d'adaptation du territoire au risque. Dépassant la simple extension d'une rationalité de marché, les pratiques de responsabilisation dans ces programmes d'adaptation et de résilience apparaissent comme des combinaisons d'arrangements pratiques et de jugements moraux historiquement et socialement situés.

\section{Conclusion}

Au terme de cet exposé, la résilience apparait donc d'abord comme un objet difficile à situer dans les programmes s'en revendiquant, et nécessairement articulé à d'autres logiques qui contribuent à lui donner son sens politique. Dans les programmes d'adaptation et de résilience portés par l'Etat sénégalais et la Banque mondiale à Dakar, la responsabilisation des populations est d'abord morale et désigne un ou des «coupables » du risque à travers un récit pointant la construction de l'habitat informel dans des zones inondables. En pratique, cette forme de responsabilisation collective se traduit en évictions ou en déplacements de ménages, dont la prise en charge se fait au cas par cas, en fonction de leur position dans les calculs des hydrologues et topographes en charge de la délimitation des ouvrages de drainage. Cette première forme prise par la mise en place d'une « résilience urbaine ${ }^{34}$ associant planification et construction d'infrastructures d'adaptation du territoire, et culpabilisation collective entrainant un traitement individuel des populations impactées par le projet, semble s'inscrire dans une vision de la résilience comme «but à atteindre » dans laquelle la ville est considérée comme une entité ou un système physique adaptable ${ }^{35}$ (Béné, et al., 2017). La rationalité dominante ici

\footnotetext{
${ }^{33}$ Même si, à ce niveau, la responsabilité collective des habitants des quartiers informels dans les inondations est assez largement discutée (la responsabilité peut en être attribuée à l'Etat pour n'avoir pas réagi à temps par exemple). La responsabilité collective concernant la préservation des ouvrages et la gestion des déchets est, elle, largement promue en revanche, la construction d'ouvrage de drainage étant perçue comme la seule mesure efficace jusqu'à présent pour permettre la mise hors d'eau durable des zones inondées.

${ }^{34}$ Selon les termes d'une employée de la Banque Mondiale (08/09/2016).

${ }^{35}$ Les clarifications institutionnelles qui y sont associées comme le transfert de la gestion de certains ouvrages en fonction de leur importance vers l'Office National d'Assainissement du Sénégal (ONAS), accompagné d'un système de financement adéquat, visent elles aussi principalement à favoriser la pérennité des infrastructures de drainage déjà mises en place. 
s'exprime moins par un contrôle indirect sur les comportements des populations que par l'exercice direct du pouvoir régalien à disposer des hommes et des choses sur le territoire de l'Etat par le recours à la force si nécessaire ${ }^{36}$, privant les ménages cibles de toute capacité d'action sur le risque.

A l'inverse, les pratiques de mobilisation et de sensibilisation des « communautés » participent de l'extension d'une forme de gouvernmentalité parmi les habitants continuant à habiter les communes situées en zone inondable. D'abord, les ouvrages de drainage se sont imposés comme des référentiels incontournables de la lutte contre les inondations dans ces communes, en cela qu'ils sont considérés par de nombreux acteurs locaux, s'étant pour certains d'entre eux historiquement engagés dans le traitement de ces dernières, comme les premières actions efficaces et durables entreprise par l'Etat depuis le surgissement du problème dans ces quartiers ${ }^{37}$. De plus, la logique portée par ces programmes d'adaptation est promue à travers la constitution de plusieurs dispositifs d'organisation des populations et de communication autour de ces ouvrages et de leurs gestions. Leurs riverains sont incités à s'engager pour la réduction du risque d'inondation par le biais de l'entretien de ces ouvrages et par l'encouragement à la « transformation des comportements » vis-à-vis de la gestion des déchets et des eaux usées. Sans préjuger de l'efficacité de ces dispositifs de sensibilisation sur l'ensemble des processus de subjectivation des habitants de la banlieue vis-à-vis du risque, il est indéniable que des programmes tels que le PROGEP ou le projet PIS ont transformé la manière de penser la gestion du risque au niveau local et conduit des collectifs d'acteurs parmi les populations des communes sinistrées à promouvoir cette pensée. La mobilisation des populations et "l'accroissement de leur résilience » produit ici deux types d'effets : au niveau des populations et des acteurs engagés dans la lutte contre les inondations, elle recentre la question du risque autour des projets portés par l'Etat, qui en était relativement absent avant 2005 ; au niveau des promoteurs de ces programmes, elle produit une justification commode à l'absence d'action globale concernant l'assainissement et la gestion des déchets dans ces quartiers, pourtant perçue comme l'une des causes majeures des inondations.

Foucault théorise la gouvernementalité comme une notion permettant d'étudier «la capacité de l'individu « autonome » à se contrôler et le lien entre cette capacité et les formes du pouvoir politique et de l'exploitation économique » (Lemke, 2004, p.20). Dans cette perspective, la gouvernementalité néolibérale trouve son essor dans les pays capitalistes occidentaux dans les années 1980 comme une forme d'exercice du pouvoir redéfinissant les rapports de l'Etat (lui-même définis comme une « technique de gouvernement» (Foucault, 1984, p. 728)) à des "populations » perçus désormais comme des sujets autonomes qu'il s'agit de responsabiliser (Foucault, 1994). Pour ancré qu'il soit, ce mode de gestion des «populations » basé sur une forme spécifique de responsabilité individuelle, s'est depuis globalisé selon différentes voix et en s'adaptant systématiquement aux particularismes locaux (Peck et al., 2009). Mettant à jour les liens très forts entre la gouvernementalité néolibérale et le concept de résilience tel qu'il est employé dans les politiques publiques de différents pays, Jonathan Joseph (2013 b.) fait cependant valoir le caractère non hégémonique du concept aussi bien que de la forme de pouvoir à laquelle il l'associe. D'une part les pays dans lesquels s'appliquent les programmes de réduction des risques des institutions internationales comme l'ONU ou la Banque Mondiale ne sont pas des « sociétés libérales avancées » ce qui implique une forme d'organisation des pouvoirs qui rend plus incertaine l'application de la notion de gouvernementalité néolibérale comme gouvernement des conduites par la responsabilisation individuelle (Joseph, 2009) A la place, la gouvernementalité serait dirigé vers les Etats principalement comme un moyen de les responsabiliser tout en les gardant sous contrôle par le « monitoring » exercé par ces institutions (Joseph, 2016).

Dans le sillage de ces travaux, il semble que la notion de résilience ne soit pas utilisée dans les programmes s'en revendiquant dans la banlieue de Dakar pour renforcer la responsabilité individuelle

\footnotetext{
${ }^{36}$ En dernière instance, c'est bien la mise en demeure de quitter leur domicile qui pousse les ménages réticents à accepter la négociation proposée par le sous-préfet.

37 Dans la commune de Djiddah Thiaroye Kao par exemple, les ouvrages de drainage sont devenus les points autour desquels s'organisent les pompages des eaux mis en place par la municipalité, les sapeurs-pompiers, ou les comités locaux de lutte contre les inondations dans les quartiers qui restent inondés durant la saison des pluies. 
vis-à-vis du risque, mais bien vis-à-vis des ouvrages promus par l'Etat pour atténuer ce risque. Cette responsabilisation ne signe pas pour autant un transfert des responsabilités de l'Etat vers les populations mais bien l'apparition dans ces quartiers de dispositifs nouveaux de mitigation et de gestion des inondations dont la responsabilité est d'emblée partagée ${ }^{38}$, sur le modèle de nombreux projets de développement urbain depuis au moins le début des années 1980 (Jaglin, 2005). Cet aspect est néanmoins à prendre au sérieux et appelle plusieurs remarques. D'une part cette responsabilité partagée n'est assurée que tant que l'ADM continue de jouer son rôle de coordination entre les différents acteurs impliqués (populations, municipalités, services de curages des canaux etc.) et sous réserve que l'Office Nationale de l'Assainissement du Sénégal (ONAS) et les municipalités parviennent à en assurer l'entretien quotidien après la fin du projet ${ }^{39}$. S'il n'est pas neuf, ce modèle de financement continue de faire dépendre les Etats et leurs capacités d'action des financements alloués «par projet» par la Banque Mondiale. Foncièrement néolibéral, il hérite des plans d'ajustement structurels entamés dans les années 1980 (Osmont, 1995) mais trouve une justification nouvelle dans la notion de résilience en insistant sur la nécessité de ne renforcer que les capacités d'adaptation dans un contexte instable, alors même que ce système de financement participe grandement à la production de l'instabilité. Cet atavisme dans les modes de financement ne doit cependant pas cacher un certain «retour» de l'Etat Sénégalais et des institutions internationales dans la planification et dans la construction d'infrastructures urbaines, sensible depuis le début des années 2000, et illustré ici par la forme d'opérationnalisation de la résilience urbaine promue par le PROGEP. Certes ancré politiquement (Melly, 2013), ce réinvestissement de la ville semble répondre à des rationalités et des imaginaires bien différents de ceux mis en exergue par l'hypothèse de continuité du régime de pouvoir défendue par les analyses de la gouvernementalité néolibérale (Samuel, 2017). Enfin, ce réinvestissement des quartiers par l'Etat, sur la base d'une responsabilisation des habitants des quartiers informels, et les réactions d'adhésion, de réappropriation et parfois de défiance observées chez certains des acteurs actuels des communes concernées par le projet, mettent l'accent à contrario sur l'abandon relatif dont ont souffert ces quartiers précédemment. Le récit du PROGEP présenté en partie 2 apparait en effet antérieure à la reprise de la résilience par les politiques internationales et s'ancre dans des représentations morales et politiques qui continuent de renvoyer les quartiers précaires, à priori coupables, à la menace et à la désorganisation socio-spatiale. Si ces dynamiques s'articulent souvent au néolibéralisme (Perlman, 2010), elles ne sauraient en être le produit (Faye, Thioub, 2003 ; Deboulet, 2016) plaidant pour une meilleure appréhension de ces articulations au sein des politiques urbaines (Morange, 2015).

\section{Bibliographie}

BAYART J-F., Le gouvernement du monde. Une critique politique de la globalisation, Fayard, Paris, 2004.

BERnSTEIN, S. F., The Compromise of Liberal Environmentalism, New York, Columbia University Press, 2001.

BÉNÉ C., MEHTA L., MCGRANAHAN G., CANNON T., GUPTE J., TANNER T., "Resilience as a policy narrative: potentials and limits in the context of urban planning", Climate and Development, 2016 [En ligne] DOI: $10.1080 / 17565529.2017 .1301868$

BIERMANN M., HILLMER-PEGRAM K., NOEL KNAPP C., HUM R. E., “Approaching a Critical Turn? A Content Analysis of the Politics of Resilience in Key Bodies of Resilience Literature", Resilience: International Policies, Practices and Discourses, Vol. 4, n² 2, 2015 [En ligne] DOI: 10.1080/21693293.2015.1094170.

BLOT J., SPIRE A., « Déguerpissements et conflits autour des légitimités citadines dans les villes du Sud », L'Espace Politique [En ligne], $22 \mid 1$, 2014. [consulté le 01 mai 2017. URL : http://espacepolitique.revues.org/2893 ; DOI : 10.4000/espacepolitique.2893]

\footnotetext{
${ }^{38}$ A l'inverse des actions locales de lutte contre les inondations précédentes qui étaient principalement de la responsabilité des habitants eux-mêmes, organisés en grande partie sans l'aide de l'Etat à proprement parler.

${ }^{39}$ Ce transfert de responsabilité de l'ADM vers ces acteurs fait partie de la clarification des responsabilités institutionnelles mise en place par le PROGEP et doit s'accompagner d'un outil permettant à l'ONAS de bénéficier de ressources pérennes qui était en cours de construction lors de l'enquête. 
CHANDLER D., "Resilience and Human Security: The Post-interventionist Paradigm", Security Dialogue 43, n 3, p. $213-229,2012$.

CISSÉ O., SÈYE M., "Flooding in the suburbs of Dakar: impacts on the assets and adaptation strategies of households or communities", Environment and Urbanization, vol. 28, n 1, p. 183-204, 2015.

COMFORT L. K., BOIN A., DEMCHAK C., Designing Resilience. Preparing for Extreme Events, Pittsburgh, University of Pittsburgh Press, 2010.

DEBOULET A. (ed.), Repenser les quartiers précaires, Agence Française de Développement, Paris, 2016.

DELEUZE G., Pourparlers. 1972-1990, Éditions de Minuit, Paris, 1990.

DJAMENT-TRAN G., LE BLANC A., LHOMME S., RUFAT S., REGHEZZA-ZITT M., « Ce que la résilience n'est pas, ce qu'on veut lui faire dire ». Consultable en ligne et consulté le 20/04/2017: [https://halshs.archivesouvertes.fr/hal-00679293/document], 2011.

EVANS B., REID J., "Exhausted by resilience: response to the commentaries", Resilience: International Policies, Practices and Discourses, 2015, [En ligne] DOI: 10.1080/21693293.2015.1022991

FAYE O., THIOUB I., « Les marginaux et l'État à Dakar », Le Mouvement Social, Vol. 3, nº 204, p. 93-108, 2003.

FELLI R., «Adaptation et résilience : critique de la nouvelle éthique de la politique environnementale internationale », Ethique publique [En ligne], Vol. 16, nº1, 2014.

FOUCAULT M., «L'éthique de soi comme pratique de liberté », in. Dits et écrits, T. IV, p. 728-729, 1984.

FOUCAULT M., « La “gouvernementalité”», in. Dits et Ecrits, T. III, p. 635-657, 1994.

FREDERICKS R., « Dakar en proie au chaos. Politique et culture des ordures ménagères”, in. DIOUF M., FREDERICKS R. (dir.), Les arts de la citoyenneté au Sénégal. Espaces contestés et civilités urbaines, Karthala, Paris, 2013.

HACHE E., (a), « Néolibéralisme et responsabilité », Raisons politiques, Vol. 4, n²8, p. 5-9, 2007.

HACHE E., (b), « La responsabilité, une technique de gouvernementalité néolibérale ? » Raisons politiques, Vol. 4, n² 28, p. $49-65,2007$.

HANNERZ U., "Being there... and there... and there! Reflexion on multi-site ethnography", Ethnography 4 (2), p. 229 244, 2003.

JAGLIN S., « La participation au service du néolibéralisme ? Les usagers dans les services d'eau en Afrique subsaharienne », in. BACQUE M-H, REY H., SINTOMER Y, (dir.), Gestion de proximité et démocratie participative, La Découverte « Recherches », p. 271-291, 2005

JOSEPH J., "Governmentality of What? Populations, States and International Organisations", Global Society, Vol 23., p. $413-427,2009$.

JOSEPH J., (a) "Resilience as embedded neoliberalism: a governmentality approach”, Resilience, Vol. 1, n 1, p. $38-52$, 2013.

JOSEPH J., (b), "Resilience in UK and French Security Strategy: An Anglo-Saxon Bias?”, Politics, Vol. 33, p. 253 - 264 , 2013.

JOSEPH, J., « Governing through Failure and Denial: The New Resilience Agenda”, Millenium: Journal of International Studies, p. $1-21,2016$

KRIEGER K., "Resilience and Risk Studies." In. Routledge Handbook of Risk Studies. Routledge, London, p. 335 - 343. 2016.

LECLERCQ R., "The politics of risk policies in Dakar, Senegal”, International Journal of Disaster Risk Reduction, 2017.

LEMKE T., « « Marx sans guillemets » : Foucault, la gouvernementalité et la critique du néolibéralisme. Traduit de l'anglais par Marc Chemali », Actuel Marx, Vol.2, n 36, p. 13-26, 2004.

MARCUS G. E., «Ethnography in/of the world system: The emergence of multi-sited ethnography”, Annual Review of Anthropology, Vol. 24, p. 95 - 117, 1995.

MEEROW S., NEWELL. J. P., "Urban resilience for whom, what, when, where, and why?" Urban Geography, 2016 [En ligne] DOI:10.1080/02723638.2016.1206395.

MEEROW S., NEWELL. J. P., STULTS M., "Defining urban resilience. A review”, Landscape and Urban Planning, Vol. 147, p. $38-49,2016$ 
MELLY C., "Ethnography on the road: Infrastructural vision and the unruly present in contemporary Dakar", Africa, Vol. $83, n^{\circ} 3$, p. 385-402, 2013

MORANGE M., "Street trade, neoliberalisation and the control of space: Nairobi's Central Business District in the era of entrepreneurial urbanism", Journal of Eastern African Studies, 2015, [En ligne] DOI: 10.1080/17531055.2015.1018407.

OSMONT A., La Banque mondiale et les villes. Du développement à l'ajustement, Karthala, Paris, 1995

PECK P., THEODORE N., BRENNER N., "Neoliberal urbanism: Models, Moments, Mutations", Review of International Affairs, Vol. 29, n 1, p. 49-66, 2009.

PERLMAN J., Favela. Four decade living in the edge of Rio de Janeiro, Oxford University Press, Oxford, 2010.

PUGH J., « Resilience, complexity and post-liberalism », Area, 46.3, p. 313 - 319, 2014.

REID J., "The disastrous and debased subject of resilience", Development Dialogue, n58, p. 67 - 81, 2012.

SAMUEL B., «Planifier en Afrique », Politique africaine, Vol. 1, n 145, p. 5-26, 2017

SANE Y., «La décentralisation au Sénégal, ou comment réformer pour mieux maintenir le statu quo », Cybergeo : European Journal of Geography [En ligne], Espace, Société, Territoire, document 796, 2016, consulté le 29 septembre 2017. URL : http://cybergeo.revues.org/27845

SCHAER C, THIAM M. D., NYGAARD I., "Flood management in urban Senegal: an actor-oriented perspective on national and transnational adaptation interventions", Climate and Development, 2017 [En ligne] DOI: $10.1080 / 17565529.2017 .1291405$

SYLLA I., Les collectivités locales face au défi numérique : le cas des communes d'arrondissement de Dakar, soutenue le 12 juin 2009 à l'Université Cheikh Anta Diop de Dakar, 446 pages, Thèse en Cotutelle avec l'Université de Toulouse Le Mirail, co-dirigée par Pape Sakho et Alioune Kane (Sénégal) et Emmanuel Eveno (France), 2009

TEDLOCK B., "From Participant Observation to the Observation of Participation: The Emergence of Narrative Ethnography", Journal of Anthropological Research, Vol. 47, n¹, p. 69-94, 1991.

THIAM M. D., Le syndrome des inondations au Sénégal, Presses Universitaires du Sahel, Dakar, 2011.

ULLBERG S., «Ethnographier la mémoire des catastrophes. Terrain translocal a Sante $\mathrm{Fe}$ (Argentine)», in. LANGUMIER J., REVET S., (ed.) Le gouvernement des catastrophes, Karthala, Paris, 2013.

WELSH M., "Resilience and responsibility: governing uncertainty in a complex world", The Geographical Journal, Vol. $180, \mathrm{n}^{\circ} .1$, p. $15-26,2014$. 